\title{
Technical pearls in lymphatic supermicrosurgery
}

\author{
Takumi Yamamoto* , Nana Yamamoto, Takashi Kageyama, Hayahito Sakai, Yuma Fuse, Kanako Tsuihiji, Reiko Tsukuura
}

Department of Plastic and Reconstructive Surgery, National Center for Global Health and Medicine, Tokyo, Japan.

\begin{abstract}
Lymphedema is becoming a major public issue with improvement of cancer survival rate, as the disease is incurable and progressive in nature, and the number of cancer survivor with lymphedema is increasing over time. Surgical treatment is recommended for progressive lymphedema, especially when conservative therapies are ineffective. Among various lymphedema surgeries, supermicrosurgical lymphaticovenular anastomosis (LVA) is becoming popular with its effectiveness and least invasiveness. There are many technical knacks and pitfalls in LVA surgery. In preoperative evaluation, indocyanine green lymphography is recommended for considering indication and incision sites. Intraoperatively, intravascular stenting method, temporary lymphatic expansion maneuver, fieldrotating retraction, and several navigation methods are useful. The most important postoperative care is immediate compression after LVA surgery. Compression is critical to keep lymphatic pressure higher than venous pressure, allowing continuous lymph-to-venous bypass flow. These technical pearls should be shared with lymphedema surgeons for better lymphedema management.
\end{abstract}

Keywords: lymphedema, microsurgery, supermicrosurgery, cancer, lymphaticovenular anastomosis, bypass

\section{Introduction}

Lymphedema is an edematous disease because of abnormal lymph circulation, and can be divided into primary and secondary lymphedema (1-3). Primary lymphedema is lymphedema other than secondary lymphedema, including genetic disorders such as Milroy disease $(1,4)$. Secondary lymphedema develops after damage to the lymphatic system by such as trauma, lymph node dissection, infection, and radiation (2-5). With improvement of cancer survival rate, increasing number of cancer survivors suffer from lymphedema. Since lymphedema is incurable and progressive in nature, lymphedema management is becoming a major public health issue to be solved.

\section{Treatment for lymphedema}

Conservative treatments are mainstays of lymphedema treatment. The most important conservative therapy is compression treatment with either bandage or garments (1-3). Pressure gradient should be considered when bandage is applied. Therefore, appropriate bandaging requires specialized training. Garments specialized for lymphedema is recommended for most lymphedema cases. Other conservative therapies include skin care, appropriate exercise under compression therapy, and manual lymph drainage.

Even with rigorous conservative treatments, most lower extremity lymphedema cases and some upper extremity lymphedema cases cannot be controlled to stop progression of the disease. For such progressive intractable lymphedema refractory conservative treatments, surgical interventions are considered $(3,6-10)$.

\section{Lymphedema surgeries}

Surgical treatments for lymphedema are largely classified into debulking surgery and physiologic reconstructive surgery $(3,8,11)$. Debulking surgery aims to directly reduce volume by removing lymphedematous tissue; surgical resection and liposuction are applied $(3,11)$. Physiologic reconstructive surgery includes lympholymphatic bypass, lymphovenous bypass, and lymphatic tissue transfer (3,6-9,12-14). Among various lymphedema surgeries, supermicrosurgical lymphaticovenular anastomosis (LVA) is the least invasive surgery effective for progressive lymphedema $(7,8-10)$.

\section{Lymphaticovenular anastomosis (LVA)}

LVA surgery is a kind of lymphovenous bypass surgery, in which a lymph vessel is anastomosed to a nearby venule or a small vein $(6-10,11-15)$. In other lymphovenous bypass surgeries, lymph vessel or node is inserted into or attached to a vein, and tissues other than the endothelium are exposed to venous blood, which 


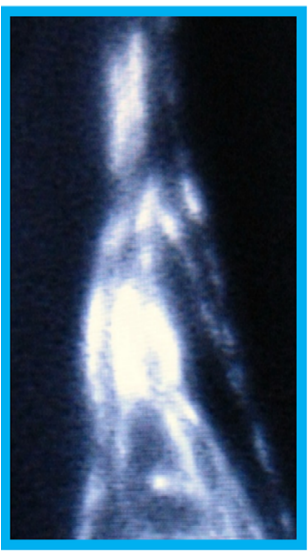

Linear

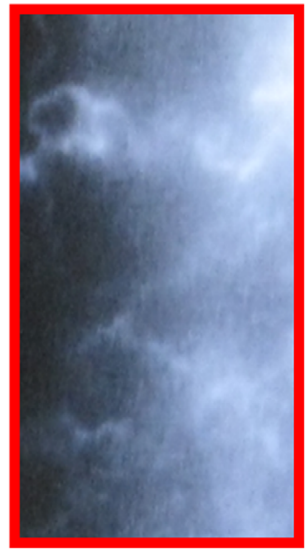

Reticular

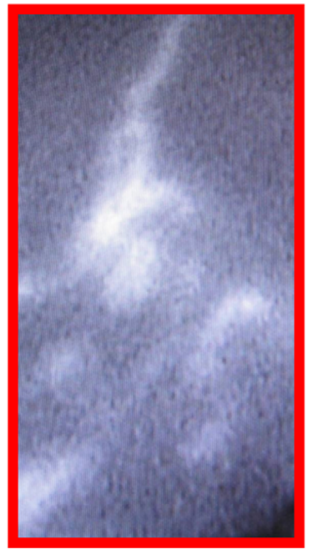

Splash

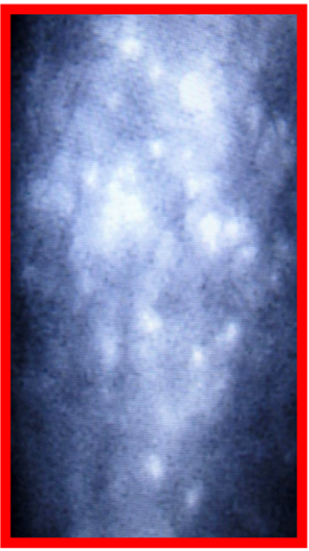

Stardust

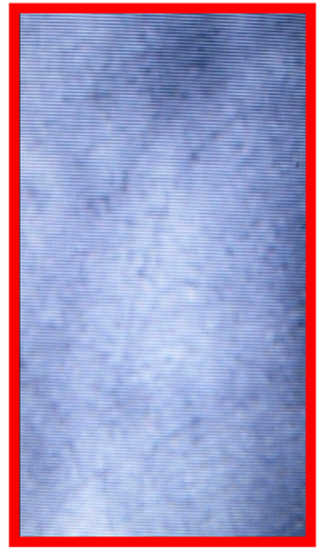

Diffuse

Figure 1. Indocyanine green lymphography findings. Normal lymphography findings is Linear pattern, whereas abnormal findings are Reticular, Splash, Stardust, and Diffuse patterns.

results in clot formation; thrombosis rate is high, and even deep vein thrombosis and pulmonary embolism have been reported $(3,6,15)$. On the other hand, a lymph vessel is anastomosed to a vein in an intimato-intima coaptation manner in supermicrosurgical LVA. The lumen of anastomosis site is covered by the endothelium, which prevents thrombosis even when venous blood contacts the anastomosis site. LVA can be performed under local infiltration anesthesia via a small skin incision, and no serious postoperative complication is reported $(6-9,12-14)$.

Although LVA allows minimally invasive surgical treatments effective for compression-refractory progressive lymphedema, sophisticated microsurgical technique called supermicrosurgery is required $(6,7,8,16)$. Supermicrosurgery is a microsurgical technique manipulating vessels with diameter of $0.5 \mathrm{~mm}$ or smaller, whereas conventional microsurgery deals with 1-2 mm vessels. A supermicrosurgeon has to master the feeling of sensation of a tip of a 50 micron needle for secure suturing $(6,7,17)$. Understanding physiology, pathophysiology, and mechanism of lymphedema and LVA is also a key to successful management of lymphedema with appropriate perioperative management.

\section{Preoperative management}

Preoperative evaluation of lymph flow is critical for surgical indication and selection of incision sites. Indocyanine green (ICG) lymphography is the most useful imaging modality for lymph circulation. Near-infrared fluorescent images are obtained after intradermal or subcutaneous ICG injection at 2 phases; at an early transient phase observation immediately after ICG injection, and at a late plateau phase observation 2-72 hours after injection $(4,5,18,19)$. This dual-phase ICG lymphography is called dynamic
Table 1. ICG lymphography stage

\begin{tabular}{ll}
\hline Stage & ICG lymphography findings \\
\hline Stage 0 & Linear pattern only (No DB pattern) \\
Stage I & Linear pattern + Splash pattern* \\
Stage II & Linear pattern + Stardust pattern (1 region) $)^{* *}$ \\
Stage III & Linear pattern + Stardust pattern (2 regions) $)^{* *}$ \\
Stage IV & Linear pattern + Stardust pattern (3 regions)** \\
Stage V & Stardust and/or Diffuse pattern (No linear pattern) \\
\hline
\end{tabular}

* Splash pattern is usually seen around the axilla/groin. ** Upper/ lower extremity is divided into 3 regions; the upper-arm/thigh, the forearm/lower-leg, and the hand/foot. DB: dermal backflow; ICG: indocyanine green.

ICG lymphography (18). ICG lymphography findings are classified into Linear, Reticular, Splash, Stardust, and Diffuse patterns (Figure 1) (5,18). At a transient phase, Linear and/or Reticular patterns are seen. At a plateau phase, Linear, Splash, Stardust, and/or Diffuse patterns are seen. Linear pattern is a normal finding, representing collecting lymph vessels' flows. The other patterns represent abnormal findings called dermal backflow (DB). According to dynamic ICG lymphography findings, pathophysiological stage is determined, and recommended LVA sites are decided.

Based on visibility of Linear pattern and extension of DB patterns, ICG lymphography stage is determined (Table 1) (19,20). ICG stage consists of stage 0 (no lymphedema), stage I (subclinical lymphedema), stage II (early lymphedema), and stage III/IV/V (progressed lymphedema). LVA is well recommended for lymphedema staged as ICG stage II/III/IV (5$10,13,14,16)$. LVA is considered as a prophylactic treatment for stage I subclinical lymphedema; LVA reduce progression risk of subclinical lymphedema from $40 \%$ to $0-5 \%(2,3,7)$. LVA can be performed for stage $\mathrm{V}$ lymphedema, but success rate to reduce lymphedematous volume is $30-50 \%$. Vascularized lymph node transfer is recommended for stage $\mathrm{V}$ 
lymphedema $(6,8,11)$.

Regarding surgical sites for LVA, overlapping region is the most recommended. In overlapping regions, Linear pattern is seen at a transient phase, and DB pattern is seen at a plateau phase. Lymph vessels in overlapping regions are mild-moderately sclerotic affected ones which should be salvaged with LVA, and usually have high lymph flows $(5,10,16,20)$. Using lymph vessels in overlapping regions, effective bypass effects can be expected. Lymph vessels in Linear pattern represents intact lymph flows, which should be preserved and not recommended for LVA. Lymph vessels in Diffuse pattern are usually severely sclerotic, not suitable for LVA $(8,16,20)$.

\section{Various techniques for lymphatic supermicrosurgery}

Since lymph vessels used in LVA are approximately $0.3-0.5 \mathrm{~mm}$ in external diameter, supermicrosurgery is necessary to perform secure LVA. To address this technical challenge, various methods can be used. A nylon thread can be used as a stent to keep vessels' lumen open during anastomosis; intravascular stenting (IVaS) method $(6,7,16)$. In IVaS method for LVA, several millimeter-long 3-0 to 7-0 nylon thread is inserted into a lymph vessel. IVaS method is available for all anastomotic configurations; end-to-end (EE), end-to-side (ES), side-to-end (SE), and side-to-side (SS) anastomoses $(6,12-14)$. Various direction skin retractors are useful to fix a surgical field for better view; upward retraction is useful to do LVA in a medial aspect of extremity.

For SE or SS anastomosis, temporary lymphatic expansion (TLE) maneuver is helpful for easier lymphotomy $(12,13)$. The most difficult procedure in SE or SS LVA is lymphotomy, a window creation on a side wall of a lymph vessel. TLE maneuver, proximal temporary lymph vessel cramping and distal limb massage, expands the lymph vessel, making it easier and safer to do lymphotomy. TLE is also useful to evaluate severity of lymphosclerosis. When a lymph vessel is expanded by TLE maneuver, it is less sclerotic and suitable for SE or SS anastomosis $(13,20)$. Parachute technique, untied continuous suture, is helpful to perform secure SE and SS anastomosis.

Sometimes "one" lymph vessel show 2 or more lumens inside, confirmed after transection of the vessel. This is seen more frequently in cases with past history of lymphangitis, and represents attached multiple lymph vessels because of inflammatory adhesion. These lumens can be changed to one lumen by resenting the septum-like vessels' wall; mono-canalization technique $(6,13,16)$. Mono-canalization allows easier anastomosis, with significantly less diameter discrepancy to a recipient vein; vein is larger in most cases.

Continuous lymph-to-venous flow is a key to long-term patency of LVA $(6,10,16,19)$. There are 2 important points; prevention of venous reflux and increasing lymph flow into one recipient vein. To prevent venous reflux into an anastomosis site, valvuloplasty, neo-valvuloplasty, venous-branchplasty, and valve-containing-venous grafting are useful (12-14). To increase lymph flow into a recipient vein, multiple-in-one (MIO) concept is important; multiple lymph flows are bypassed into one vein. In MIO concept, utilizing all anastomotic configuration plays an important role. Lambda-shaped anastomosis allows both proximal and distal lymph flows in one vein, in which proximal ES and distal EE anastomoses are performed (6). Sequential anastomosis diverts many lymph flows into one vein with the use of SS anastomoses. Other anastomotic combinations include, multiple SE anastomoses, all-star anastomoses, and ladder-shaped anastomoses.

\section{Postoperative management}

Immediate postoperative compression keeps anastomosis sites patent, with continuous lymph-to-venous flows via the anastomoses $(3,6,7,16)$. In a lymphedematous limb, lymphatic system is closed; obstructed in the proximal region. When the limb is compressed, lymphatic pressure becomes higher. On the other hand, venous system is open, and venous pressure stays constant when compressed. Therefore, postoperative compression increases only lymphatic pressure, which leads to lymphto-venous pressure gradient and continuous lymph flow via an anastomosis. In other microsurgical or supermicrosurgical vascular anastomosis, compression should be avoided. However, postoperative compression should be resumed immediately after operation in LVA surgery.

\section{Conclusions}

Supermicrosurgical LVA is minimally invasive surgical treatment effective for progressive lymphedema. ICG lymphography is useful to consider indication and skin incision sites of LVA. Various techniques help a surgeon to do this challenging supermicrosurgery. Immediate postoperative compression is recommended for better clinical results.

\section{Acknowledgements}

Preparation of this manuscript was supported in part by National Center for Global Health and Medicine (NCGM) biobank fund (29-2004).

\section{References}

1. Schook CC, Mulliken JB, Fishman SJ, Grant FD, Zurakowski D, Greene AK. Primary lymphedema: clinical features and management in 138 pediatric patients. Plast Reconstr Surg. 2011; 127:2419-2431. 
2. Yamamoto T, Yamamoto N, Yamashita M, Furuya M, Hayashi A, Koshima I. Efferent lymphatic vessel anastomosis (ELVA): supermicrosurgical efferent lymphatic vessel-to-venous anastomosis for the prophylactic treatment of subclinical lymphedema. Ann Plast Surg. 2016; 76:424-427.

3. Murdaca G, Cagnati P, Gulli R, Spanò F, Puppo F, Campisi C, Boccardo F. Current views on diagnostic approach and treatment of lymphedema. Am J Med. 2012; 125:134-140.

4. Yamamoto T, Yoshimatsu H, Narushima M, Yamamoto N, Hayashi A, Koshima I. Indocyanine green lymphography findings in primary leg lymphedema. Eur J Vasc Endovasc Surg. 2015; 49:95-102.

5. Yamamoto T, Narushima M, Doi K, Oshima A, Ogata F, Mihara M, Koshima I, Mundinger GS. Characteristic indocyanine green lymphography findings in lower extremity lymphedema: the generation of a novel lymphedema severity staging system using dermal backflow patterns. Plast Reconstr Surg. 2011; 127:19791986.

6. Yamamoto T, Narushima M, Kikuchi K, Yoshimatsu H, Todokoro T, Mihara M, Koshima I. Lambda-shaped anastomosis with intravascular stenting method for safe and effective lymphaticovenular anastomosis. Plast Reconstr Surg. 2011; 127:1987-1992.

7. Yamamoto T, Narushima M, Yoshimatsu H, Seki Y, Yamamoto N, Oka A, Hara H, Koshima I. Minimally invasive lymphatic supermicrosurgery (MILS): indocyanine green lymphography-guided simultaneous multi-site lymphaticovenular anastomoses via millimeter skin incisions. Ann Plast Surg. 2014; 72:67-70.

8. Yamamoto T, Yoshimatsu H, Yamamoto N. Complete lymph flow reconstruction: a free vascularized lymph node true perforator flap transfer with efferent lymphaticolymphatic anastomosis. J Plast Reconstr Aesthet Surg. 2016; 69:1227-1233.

9. Yamamoto T, Saito T, Ishiura R, Iida T. Quadruplecomponent superficial circumflex iliac artery perforator (SCIP) flap: a chimeric SCIP flap for complex ankle reconstruction of an exposed artificial joint after total ankle arthroplasty. J Plast Reconstr Aesthet Surg. 2016; 69:1260-1265.

10. Yamamoto T, Kikuchi K, Yoshimatsu H, Koshima I. Ladder-shaped lymphaticovenular anastomosis using multiple side-to-side lymphatic anastomoses for a leg lymphedema patient. Microsurgery. 2014; 34:404-408.

11. Brorson H, Ohlin K, Olsson G, Svensson B, Svensson H. Controlled compression and liposuction treatment for lower extremity lymphedema. Lymhology. 2008; 41:5263 .

12. Yamamoto T, Yoshimatsu H, Narushima M, Seki Y,
Yamamoto N, Shim TW, Koshima I. A modified side-toend lymphaticovenular anastomosis. Microsurgery. 2013; 33:130-133.

13. Yamamoto $\mathrm{T}$, Yoshimatsu H, Yamamoto N, Narushima M, Iida T, Koshima I. Side-to-end lymphaticovenular anastomosis through temporary lymphatic expansion. PLoS One. 2013; 8:e59523.

14. Yamamoto T, Chen WF, Yamamoto N, Yoshimatsu $\mathrm{H}$, Tashiro K, Koshima I. Technical simplification of the supermicrosurgical side-to-end lymphaticovenular anastomosis using the parachute technique. Microsurgery. 2015; 35:129-134.

15. Ishiura R, Yamamoto T, Siato T, Mito D, Iida T. Comparison of lympho-venous shunt methods in rat model: supermicrosurgical lymphaticovenular anastomosis versus microsurgical lymphaticovenous implantation. Plast Reconstr Surg. 2017; 139:1407-1413.

16. Yamamoto T. Onco-reconstructive supermicrosurgery. Eur J Surg Oncol. 2019; 45:1146-1151.

17. Yamamoto T, Yamamoto N, Yamashita M, Furuya M, Hayashi A, Koshima I. Establishment of supermicrosurgical lymphaticovenular anastomosis model in rat. Microsurgery. 2017; 37:57-60.

18. Yamamoto T, Narushima M, Yoshimatsu H, Yamamoto N, Kikuchi K, Todokoro T, Iida T, Koshima I. Dynamic indocyanine green lymphography for breast cancer-related arm lymphedema. Ann Plast Surg. 2014; 73:706-709.

19. Yamamoto T, Matsuda N, Doi K, Oshima A, Yoshimatsu H, Todokoro T, Ogata F, Mihara M, Narushima M, Iida $\mathrm{T}$, Koshima I. The earliest finding of indocyanine green lymphography in asymptomatic limbs of lower extremity lymphedema patients secondary to cancer treatment: the modified dermal backflow stage and concept of subclinical lymphedema. Plast Reconstr Surg. 2011; 128:314e-321e.

20. Yamamoto T, Yamamoto N, Yoshimatsu H, Narushima M, Koshima I. Factors associated with lymphosclerosis: an analysis on 962 lymphatic vessels. Plast Reconstr Surg. 2017; 140:734-741.

Received July 29, 2019; Revised January 28, 2020; Accepted January 29, 2020.

Released online in J-STAGE as advance publication February 2,2020 .

*Address correspondence to:

Takumi Yamamoto, Department of Plastic and Reconstructive Surgery, National Center for Global Health and Medicine, 1-21-1 Toyama Shinjuku-ku, Tokyo 162-8655, Japan.

E-mail: tyamamoto-tky@umin.ac.jp 